Original

\title{
Mortalidad por complicaciones médicas y quirúrgicas, impacto de la crisis y gasto sanitario en España, 2002-2013
}

\author{
Andreu Nolasco ${ }^{a}$, Eva M. Vicent-Castellób ${ }^{\text {, Pamela Pereyra-Zamora }}{ }^{a}$, Pablo Caballero-Pérez ${ }^{a, *}$ \\ y Joaquín Moncho \\ a Departamento de Enfermería Comunitaria, Medicina Preventiva y Salud Pública, e Historia de la Ciencia, Universidad de Alicante, Alicante, España \\ ${ }^{\mathrm{b}}$ Formación para el Empleo, Ayuntamiento de Albal, Albal (Valencia), España
}

\section{INFORMACIÓN DEL ARTÍCULO}

\section{Historia del artículo:}

Recibido el 29 de marzo de 2018

Aceptado el 20 de julio de 2018

On-line el $\mathrm{xxx}$

\section{Palabras clave:}

Mortalidad tratable

Crisis económica

Gasto sanitario

\begin{abstract}
R E S U M E N
Objetivo: Describir la evolución de los riesgos de mortalidad por complicaciones debidas a la atención médica o la cirugía entre los periodos anterior (2002-2007) y posterior (2008-2013) al inicio de la crisis económica, en España y por comunidades autónomas, y analizar la relación entre los cambios en los riesgos de muerte y el impacto socioeconómico de la crisis y la variación del gasto sanitario.

Método: Estudio ecológico basado en tasas de mortalidad estandarizadas por edad, índice sintético de vulnerabilidad como indicador socioeconómico y variación del gasto sanitario como indicador del gasto en salud. Se estimó el riesgo relativo de muerte entre periodos con modelos de regresión de Poisson. Resultados: El número de muertes aumentó para España en el periodo estudiado. Aunque la relación entre el incremento en la inversión pública en salud y la disminución de la mortalidad por esta causa no ha quedado claramente demostrada, sí ha podido determinarse que aquellas comunidades autónomas con menor incremento del gasto sanitario presentaron mayores tasas que el resto a lo largo de todo el periodo, y que las más vulnerables a la crisis y con menor incremento del gasto presentaron un mayor incremento de riesgo de muerte entre periodos.

Conclusión: Dado el incremento de las muertes debidas a fallos evitables del sistema, es necesario seguir investigando sobre esta causa de mortalidad.

(C) 2018 SESPAS. Publicado por Elsevier España, S.L.U. Este es un artículo Open Access bajo la licencia CC BY-NC-ND (http://creativecommons.org/licenses/by-nc-nd/4.0/).

\section{Mortality due to medical and surgical complications, economic crisis and health spending in Spain, 2002-2013}

\section{A B S T R A C T}

Objective: To describe the evolution of mortality risks for complications due to medical care or surgery between the periods prior to (2002-2007) and after (2008-2013) the beginning of the economic crisis for Spain and by autonomous region, and to analyse the relationship between the changes in the risks of death and the socioeconomic impact of the crisis and the variation in health spending.

Method: Ecological study based on age-standardized mortality rates, synthetic index of vulnerability as a socioeconomic indicator and variation in health expenditure as an indicator of health expenditure. The relative risk of death between periods was estimated with Poisson regression models.

Results: The number of deaths increased for Spain in the period studied. Although the relationship between the increase in public investment in health and the decrease in mortality due to this cause has not been clearly demonstrated, it was possible to determine that the autonomous regions with the lowest increase in health expenditure had rates higher than the rest throughout the period, and that the most vulnerable to the crisis and with the lowest increase in spending presented the greatest increase in the risk of death between the periods.

Conclusions: Given the increase in these deaths, due to avoidable failures of the system, it is necessary to continue investigating this cause of mortality.

(c) 2018 SESPAS. Published by Elsevier España, S.L.U. This is an open access article under the CC BY-NC-ND license (http://creativecommons.org/licenses/by-nc-nd/4.0/).

\section{Introducción}

Como parte de las muertes evitables, la mortalidad tratable hace referencia a aquellas que no deberían haberse producido, o podrían haberse retrasado, y que son conse-

* Autor para correspondencia.

Correo electrónico: pablo.caballero@ua.es (P. Caballero-Pérez). 
y directamente con la intervención sanitaria, ya sea con el acceso a la asistencia sanitaria, consejo, diagnóstico o tratamiento.

La calidad de la asistencia sanitaria y el acceso equitativo a ella deben considerarse como objetivos prioritarios de las políticas de salud. Es en este marco en el que la mortalidad tratable ha sido considerada como un indicador que permite evidenciar las potenciales debilidades del sistema sanitario y su impacto sobre la salud de la población ${ }^{1,2}$.

Diversos estudios han demostrado una reducción en la mortalidad tratable en los países de ingresos altos, con diferencias geográficas importantes, y una asociación entre la mortalidad tratable y diferentes factores socioeconómicos de la población ${ }^{3,4}$. Aunque la reducción de estas causas de mortalidad en los países de ingresos altos se ha relacionado con la mejora de los sistemas sanitarios, no se ha obtenido una asociación clara entre los indicadores de calidad del sistema sanitario y la mortalidad evitable $^{5-7}$.

Dentro de la mortalidad tratable existe un grupo de causas que están directamente relacionadas con la calidad de la atención sanitaria prestada. Se trata de la "mortalidad por complicaciones debidas a la atención médica o cirugía». Se incluyen en este grupo las "complicaciones de dispositivos sanitarios, procedimientos quirúrgicos y médicos como causa de reacción anormal del paciente o de complicación posterior, sin mención de efecto adverso en el momento del procedimiento" ${ }^{8}$.

Estas causas se relacionan con el concepto de "efectos adversos», aunque no incluyan todos ellos. En España, según el Ministerio de Salud, Servicios Sociales e Igualdad (MSSSI), en torno al 9-12\% de los pacientes atendidos en hospitales y el 1-2\% de los atendidos en atención primaria sufre un efecto adverso, de los cuales un 50-70\% son evitables ${ }^{9}$. Según el Estudio Nacional sobre los Efectos Adversos ligados a la Hospitalización (ENEAS), publicado en 2006, un $4,4 \%$ de estos pacientes fallecieron, un $66,3 \%$ precisó procedimientos adicionales y se produjeron 3200 estancias adicionales por esta causa ${ }^{10}$. En 2011 se estimó que los efectos adversos por medicación, infección nosocomial y procedimientos quirúrgicos habían supuesto un sobrecoste de 2474 millones de euros al Sistema Nacional de Salud ${ }^{9}$.

Según el modelo explicativo de Reason (1997) sobre las causas de los efectos adversos, los fallos del sistema son más importantes que los fallos humanos en la cadena causal de un efecto adverso, pero entre los fallos humanos, los debidos a las condiciones de trabajo y en concreto a la sobrecarga asistencial son considerados los más importantes ${ }^{11}$.

En España, en la segunda mitad de 2010 y como consecuencia de la crisis económica, se iniciaron políticas de austeridad ${ }^{12}$ que afectaron entre otros al Sistema Nacional de Salud, en el que las comunidades autónomas son las encargadas de gestionar los servicios. Estas reformas, en adición al deterioro socioeconómico consecuencia del inicio de la crisis, podrían haber afectado tanto a la calidad del propio sistema como a la de los servicios prestados por sus profesionales, provocando un aumento de los efectos adversos en los servicios sanitarios y un aumento de la mortalidad por causas tratables.

El objetivo de este estudio es describir, para el conjunto de España y por comunidades autónomas, la evolución de los riesgos de mortalidad por complicaciones debidas a la atención médica o cirugía (códigos CIE 10 Y60-Y69, Y83-Y84) entre los periodos 2002-2007 y 2008-2013, antes y después del inicio de la crisis económica, así como analizar la relación de los cambios en los riesgos de muerte con indicadores socioeconómicos del impacto de la crisis y con indicadores de cambio del gasto sanitario entre periodos en las comunidades autónomas.

\section{Método}

Diseño

Estudio ecológico de mortalidad para describir la evolución de los riesgos de muerte del periodo 2002-2007 al periodo 20082013, para el conjunto de España y por comunidades autónomas. Se excluyeron las ciudades autónomas de Ceuta y Melilla.

\section{Datos de mortalidad y población}

Del Instituto Nacional de Estadística se obtuvieron los datos de las muertes ocurridas en residentes en España durante el periodo en estudio (de 2002 a 2013) con las variables sexo, edad, comunidad autónoma de residencia y causa de la muerte, y las poblaciones anuales por edad (grupos quinquenales) y sexo, procedentes de los padrones municipales continuos a fecha 1 de enero.

\section{Indicadores socioeconómicos}

Para cada comunidad autónoma se utilizó el índice sintético de vulnerabilidad (ISV). Este índice se construye a partir de la evolución de 15 indicadores desde antes del inicio de la crisis hasta después de su inicio, y puede tomar un valor estandarizado entre -2 y 2 (mínima y máxima vulnerabilidad), obtenido como media de tres índices de vulnerabilidad económica, social e inmobiliaria calculados por Méndez et al. ${ }^{13}$ para las provincias españolas. En este estudio, los indicadores han sido recalculados para cada comunidad autónoma ponderando los indicadores de sus provincias según el tamaño poblacional.

\section{Indicador de gasto sanitario}

Los datos sobre gasto sanitario total para las 17 comunidades autónomas del territorio nacional, para cada año en estudio, se obtuvieron del MSSSI ${ }^{14}$. Con esta información, tras calcular el gasto sanitario medio anual per cápita (GS) como el cociente entre el gasto total y la población residente, para cada comunidad autónoma se construyó el indicador de variación del gasto sanitario (VGS) entre periodos como el incremento porcentual del gasto medio per cápita del periodo 2008-2013 respecto al periodo 2002-2007.

\section{Agrupación de las comunidades autónomas}

Para la estimación del riesgo relativo(RR) de muerte del segundo periodo respecto del primero se agruparon las comunidades autónomas según el tercil de pertenencia en el ISV y en la VGS, de forma que el primer tercil representa la situación más favorable en ISV (menor índice de vulnerabilidad) o en VGS (mayor incremento porcentual del gasto) y el tercer tercil representa la situación menos favorable (mayor ISV, menor VGS), mientras que el tercil intermedio recoge las situaciones intermedias.

\section{Causa de muerte estudiada}

Complicaciones debidas a la atención médica o cirugía general, códigos de la Clasificación Internacional de Enfermedades,

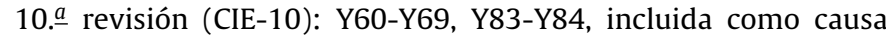
de mortalidad evitable tratable entre las propuestas por Nolte y McKee $^{1,2}$.

\section{Análisis de los datos}

El análisis de los datos se realizó para ambos sexos conjuntamente. Para el total de España, por comunidades autónomas 
Tabla 1

Poblaciones medias y defunciones para los periodos 2002-2004, 2005-2007, 2008-2010 y 2011-2013, en España y las comunidades autónomas

\begin{tabular}{|c|c|c|c|c|c|c|c|c|}
\hline & \multicolumn{2}{|c|}{ 2002-04 } & \multicolumn{2}{|c|}{ 2005-07 } & \multicolumn{2}{|c|}{$2008-10$} & \multicolumn{2}{|c|}{$2011-13$} \\
\hline & Población & Muertes & Población & Muertes & Población & Muertes & Población & Muertes \\
\hline Andalucía & 7.590 .933 & 188 & 7.961 .644 & 195 & 8.292 .039 & 181 & 8.445 .130 & 106 \\
\hline Aragón & $1232 . .396$ & 35 & 1.281 .051 & 15 & 1.339 .829 & 36 & 1.348 .272 & 27 \\
\hline Asturias, Principado de & 1.074 .371 & 9 & 1.076 .131 & 14 & 1.083.256 & 25 & 1.073 .926 & 13 \\
\hline Balears, Illes & 939.791 & 6 & 1.004 .948 & 11 & 1.091 .440 & 2 & 1.116 .679 & 2 \\
\hline Canarias & 1.884 .721 & 41 & 1.996 .688 & 41 & 2.099 .493 & 33 & 2.117 .983 & 40 \\
\hline Cantabria & 548.916 & 37 & 567.741 & 33 & 587.874 & 18 & 593.032 & 74 \\
\hline Castilla-La Mancha & 1.815 .567 & 34 & 1.934 .744 & 38 & 2.074 .262 & 80 & 2.114 .405 & 76 \\
\hline Castilla y León & 2.487.311 & 128 & 2.520 .762 & 41 & 2.560 .122 & 87 & 2.536 .035 & 88 \\
\hline Cataluña & 6.674 .635 & 123 & 7.113 .470 & 109 & 7.450 .626 & 80 & 7.563.477 & 126 \\
\hline Comunitat Valenciana & 4.446 .966 & 12 & 4.794 .795 & 51 & 5.078 .661 & 71 & 5.123 .219 & 99 \\
\hline Extremadura & 1.074 .080 & 9 & 1.086 .747 & 16 & 1.102 .458 & 18 & 1.106 .435 & 15 \\
\hline Galicia & 2.746 .483 & 14 & 2.767 .418 & 11 & 2.792 .637 & 17 & 2.775 .186 & 13 \\
\hline Madrid, Comunidad de & 5.683 .641 & 18 & 6.018 .005 & 18 & 6.372 .418 & 23 & 6.496 .089 & 37 \\
\hline Murcia, Región de & 1.263 .639 & 21 & 1.366.072 & 10 & 1.444 .869 & 21 & 1.473 .504 & 19 \\
\hline Navarra, Comunidad Foral de & 577.524 & 13 & 600.407 & 14 & 629.293 & 6 & 644.361 & 12 \\
\hline País Vasco & 2.111 .921 & 63 & 2.133 .463 & 46 & 2.169.209 & 73 & 2.192 .138 & 93 \\
\hline Rioja, La & 287.519 & 11 & 305476 & 9 & 320.539 & 3 & 322.991 & 19 \\
\hline España & 42.440 .414 & 762 & $44 . .529 .562$ & 672 & 46.489 .025 & 774 & 47.042 .862 & 859 \\
\hline
\end{tabular}

Tabla 2

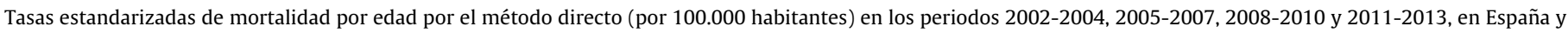
las comunidades autónomas

\begin{tabular}{|c|c|c|c|c|c|c|c|c|}
\hline & \multicolumn{2}{|c|}{$2002-2004$} & \multicolumn{2}{|c|}{$2005-2007$} & \multicolumn{2}{|c|}{$2008-2010$} & \multicolumn{2}{|c|}{ 2011-2013 } \\
\hline & TEM & IC95\% & TEM & IC95\% & TEM & IC95\% & TEM & IC95\% \\
\hline Andalucía & 0,95 & $0,81-1,09$ & 0,94 & $0,81-1,08$ & 0,81 & $0,69-0,93$ & 0,45 & $0,36-0,53$ \\
\hline Aragón & 0,80 & $0,53-1,07$ & 0,32 & $0,16-0,48$ & 0,77 & $0,52-1,03$ & 0,57 & $0,35-0,79$ \\
\hline Asturias, Principado de & 0,23 & $0,08-0,38$ & 0,33 & $0,16-0,50$ & 0,57 & $0,34-0,80$ & 0,28 & $0,13-0,44$ \\
\hline Balears, Illes & 0,26 & $0,05-0,47$ & 0,43 & $0,18-0,69$ & 0,07 & $0,00-0,16$ & 0,06 & $0,00-0,15$ \\
\hline Canarias & 1,00 & $0,69-1,32$ & 0,88 & $0,60-1,15$ & 0,63 & $0,41-0,85$ & 0,71 & $0,49-0,94$ \\
\hline Cantabria & 2,11 & $1,43-2,80$ & 1,70 & $1,12-2,28$ & 0,86 & $0,46-1,26$ & 3,46 & $2,66-4,25$ \\
\hline Castilla-La Mancha & 0,58 & $0,38-0,78$ & 0,61 & $0,41-0,80$ & 1,23 & $0,95-1,50$ & 1,11 & $0,85-1,36$ \\
\hline Castilla y León & 1,38 & $1,14-1,63$ & 0,41 & $0,29-0,54$ & 0,83 & $0,66-1,01$ & 0,77 & $0,60-0,94$ \\
\hline Cataluña & 0,64 & $0,52-0,75$ & 0,52 & $0,42-0,62$ & 0,36 & $0,28-0,43$ & 0,52 & $0,43-0,61$ \\
\hline Comunitat Valenciana & 0,10 & $0,04-0,15$ & 0,38 & $0,27-0,48$ & 0,48 & $0,37-0,60$ & 0,62 & $0,50-0,75$ \\
\hline Extremadura & 0,25 & $0,09-0,42$ & 0,42 & $0,22-0,63$ & 0,46 & $0,25-0,68$ & 0,38 & $0,19-0,58$ \\
\hline Galicia & 0,16 & $0,07-0,24$ & 0,11 & $0,04-0,18$ & 0,16 & $0,08-0,23$ & 0,12 & $0,05-0,18$ \\
\hline Madrid, Comunidad de & 0,13 & $0,07-0,19$ & 0,12 & $0,06-0,17$ & 0,14 & $0,08-0,19$ & 0,20 & $0,13-0,26$ \\
\hline Murcia, Región de & 0,68 & $0,38-0,98$ & 0,33 & $0,12-0,53$ & 0,60 & $0,34-, 86$ & 0,48 & $0,26-0,70$ \\
\hline Navarra, Comunidad Foral de & 0,73 & $0,33-1,13$ & 0,75 & $0,36-1,15$ & 0,30 & $0,06-0,55$ & 0,59 & $0,25-0,93$ \\
\hline País Vasco & 0,96 & $0,72-1,20$ & 0,67 & $0,48-0,87$ & 0,99 & $0,76-1,22$ & 1,19 & $0,95-1,44$ \\
\hline Rioja, La & 1,16 & $0,47-1,84$ & 0,90 & $0,31-1,50$ & 0,29 & $0,00-0,61$ & 1,77 & $0,96-2,57$ \\
\hline España & 0,62 & $0,58-0,66$ & 0,51 & $0,48-0,55$ & 0,55 & $0,52-0,59$ & 0,57 & $0,53,0,61$ \\
\hline
\end{tabular}

IC95\%: intervalo de confianza del 95\%; TEM: tasas estandarizadas de mortalidad.

y por agrupaciones en terciles según ISV y VGS de las comunidades autónomas se calcularon las tasas estandarizadas de mortalidad (TEM) por edad por el método directo, utilizando como población estándar la de España, y sus correspondientes intervalos de confianza del 95\% (IC95\%), considerando los subperiodos 2002-2004, 2005-2007, 2008-2010 y 2011-2013. La estimación del RR de muerte (y su IC95\%) de 2008-2013 vs. 2002-2007 se obtuvo a partir de modelos de regresión de Poisson, ajustando por edad (cuatro grupos: 0-14, 15-59, 60-74 y $\geq 75$ años) y estratificando por los terciles de agrupación de las comunidades autónomas.

\section{Consideraciones éticas}

Este estudio utiliza datos retrospectivos anónimos, no identificables, y ha sido realizado de acuerdo con la Declaración de Helsinki modificada. La legislación española no requiere consentimiento informado ni la aprobación de un Comité Ético para estos estudios.

\section{Resultados}

Entre 2002 y 2013 se produjeron 3067 muertes en ambos sexos por causas relacionadas con la atención médica o cirugía, de las cuales 1434 ocurrieron en el periodo anterior a la crisis económica (de 2002 a 2007) y 1633 en el periodo tras el inicio de la crisis económica (de 2008 a 2013) (tabla 1).

En la tabla 2 puede observarse la evolución de las TEM para España y para cada una de las comunidades autónomas durante los cuatro periodos. Para el conjunto de España no se produjeron cambios significativos entre los periodos estudiados. Por comunidades autónomas, Cantabria, Castilla y León, La Rioja, Canarias, País Vasco y Andalucía evidenciaron mayores riesgos de muerte por esta causa. La mayoría de las comunidades autónomas registraron una tendencia decreciente en el riesgo de muerte hasta 2007. A partir de 2008, coincidiendo con el inicio de la crisis, se detectó un cambio de tendencia al alza en buena parte de ellas; destacables fueron los casos de Cantabria y La Rioja en el último periodo.

La tabla 3 muestra los valores del ISV, del GS y de la VGS en los periodos 2002-2007 y 2008-2013 por comunidades autónomas, así como los terciles de pertenencia de cada una de ellas. Pueden 
Tabla 3

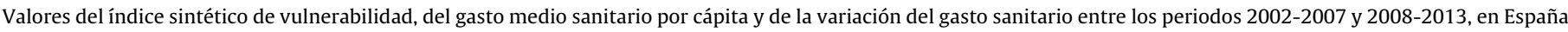
y las comunidades autónomas

\begin{tabular}{|c|c|c|c|c|c|c|}
\hline & ISV & Tercil & GS 2002-07 & GS 2008-13 & VGS $2002-07$ a $2008-13$ & Tercil \\
\hline Andalucía & 0,21 & 2 & 954,4 & 1169,0 & 22,5 & 3 \\
\hline Aragón & 0,34 & 2 & 1124,0 & 1454,0 & 29,4 & 2 \\
\hline Asturias & $-0,33$ & 1 & 1141,0 & 1531,4 & 34,2 & 1 \\
\hline Balears, Illes & 0,47 & 3 & 1020,8 & 1319,5 & 29,3 & 2 \\
\hline Canarias & 0,07 & 2 & 1112,0 & 1341,5 & 20,6 & 3 \\
\hline Cantabria & 0,13 & 2 & 1238,1 & 1434,9 & 15,9 & 3 \\
\hline Castilla-La Mancha & $-0,03$ & 2 & 1037,9 & 1396,8 & 34,6 & 1 \\
\hline Castilla y León & 0,55 & 3 & 1071,0 & 1375,1 & 28,4 & 2 \\
\hline Cataluña & 0,71 & 3 & 1040,1 & 1341,8 & 29,0 & 2 \\
\hline Comunitat Valenciana & 0,99 & 3 & 995,8 & 1274,3 & 28,0 & 3 \\
\hline Extremadura & $-0,87$ & 1 & 1127,9 & 1506,6 & 33,6 & 1 \\
\hline Galicia & $-0,56$ & 1 & 1061,5 & 1359,2 & 28,0 & 2 \\
\hline Madrid, Comunidad de & 0,00 & 2 & 936,2 & 1214,2 & 29,7 & 1 \\
\hline Murcia, Región de & 1,07 & 3 & 1046,9 & 1505,5 & 43,8 & 1 \\
\hline Navarra, Comunidad Foral de & $-0,47$ & 1 & 1176,2 & 1508,0 & 28,2 & 2 \\
\hline País Vasco & $-0,94$ & 1 & 1161,7 & 1605,3 & 38,2 & 1 \\
\hline Rioja, La & 0,53 & 3 & 1234,1 & 1409,4 & 14,2 & 3 \\
\hline España & - & & 1139,4 & 1438,6 & 26,3 & \\
\hline
\end{tabular}

GS: gasto medio sanitario por cápita; ISV: índice sintético de vulnerabilidad; VGS: variación del gasto sanitario.

observarse diferencias en el GS en función de la comunidad autónoma y del periodo analizado. Todas las comunidades autónomas incrementaron su GS del primero al segundo periodo. La Región de Murcia $(43,8 \%)$ y el País Vasco $(38,2 \%)$ fueron las que presentaron mayor VGS, y Cantabria (15,9\%) y La Rioja $(14,2 \%)$ fueron las de menor VGS. De igual modo, se detectaron diferencias en el ISV entre comunidades autónomas, oscilando entre -0,94 en el País Vasco (menos vulnerable) y 1,07 en la Región de Murcia (más vulnerable).

Las figuras 1 y 2 muestran la evolución de las TEM a lo largo de los periodos según terciles de clasificación de las comunidades autónomas. El segundo tercil del ISV presentó unas TEM superiores al resto a lo largo de todos los periodos, a excepción del último, en el que fue superado por la agrupación del tercer tercil de mayor vulnerabilidad. Las agrupaciones de comunidades autónomas y España experimentaron un incremento en los riesgos de muerte a partir de 2008, a excepción del segundo tercil del ISV, que describe una tendencia descendente. La figura 1 recoge el cambio de tendencia.

El tercer tercil de VGS mantuvo a lo largo de todo el periodo en estudio TEM superiores a las del resto de las agrupaciones (fig. 2).

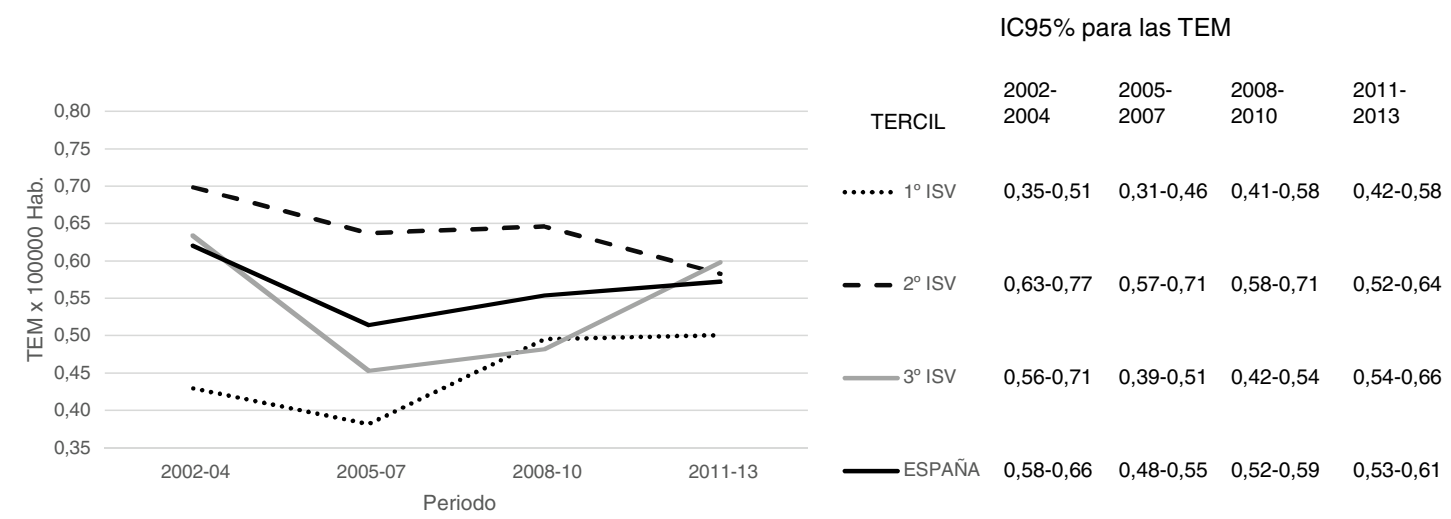

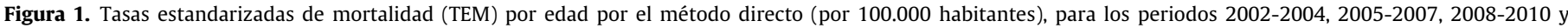
2011-2013, para España y agrupaciones de las comunidades autónomas según tercil de índice sintético de vulnerabilidad (ISV). IC95\%: intervalo de confianza del 95\%.

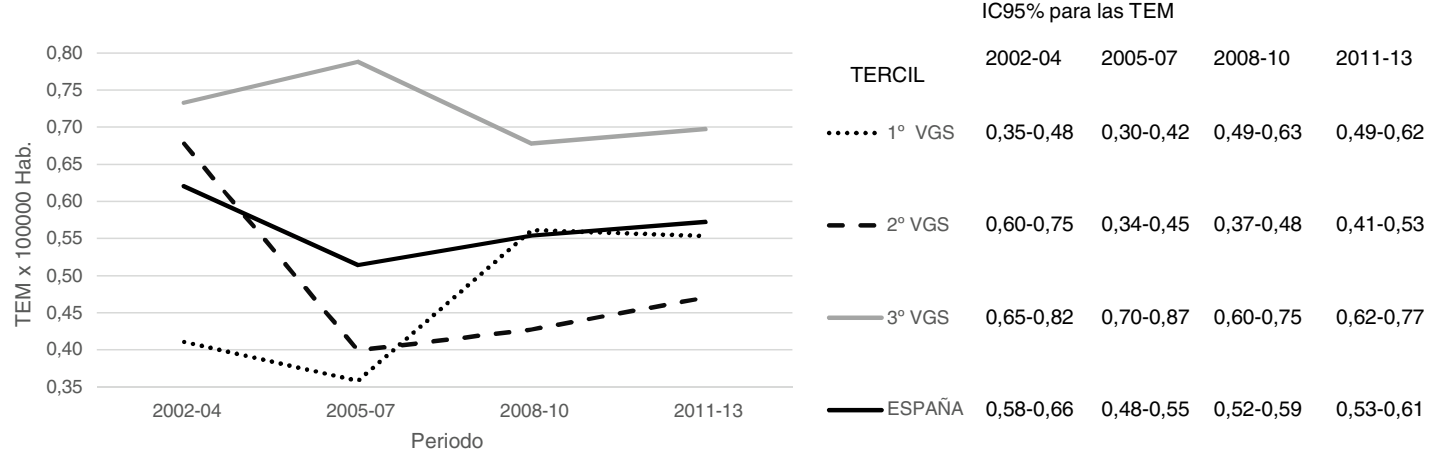

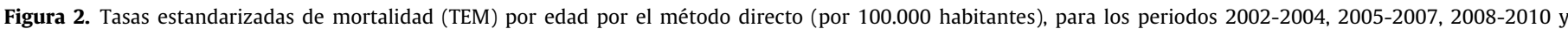
2011-2013, para España y agrupaciones de las comunidades autónomas según tercil de variación del gasto sanitario (VGS). IC95\%: intervalo de confianza del 95\%. 
Tabla 4

Riesgos relativos de muerte ajustados por edad (regresión de Poisson) del periodo 2008-2013 respecto al periodo 2002-2007

\begin{tabular}{|c|c|c|c|c|}
\hline \multicolumn{3}{|c|}{ Según terciles del ISV } & & \\
\hline Tercil & $\mathrm{RR}$ & IC95\% & & \\
\hline 1 & 1,241 & $1,038-1,484$ & & \\
\hline 2 & 0,924 & $0,832-1,025$ & & \\
\hline 3 & 1,018 & $0,906-1,143$ & & \\
\hline \multicolumn{5}{|c|}{ Según terciles de la VGS de 2002-2007 a 2008-2013 } \\
\hline Tercil & RR & IC95\% & & \\
\hline 1 & 1,457 & $1,262-1,683$ & & \\
\hline 2 & 0,854 & $0,755-0,966$ & & \\
\hline 3 & 0,907 & $0,812-1,013$ & & \\
\hline \multicolumn{5}{|c|}{ Según terciles de ISV y de la VGS conjuntamente } \\
\hline \multirow[t]{2}{*}{ RR (IC95\%) } & & & VGS & \\
\hline & Tercil 1 & & Tercil 2 & Tercil 3 \\
\hline Tercil 1 & $1,370(1,120-1,677)$ & & $0,842(0,569-1,248)$ & - \\
\hline \multicolumn{5}{|l|}{ ISV } \\
\hline Tercil 1 & $1,715(1,361-2,160)$ & & $1,179(0,813-1,709)$ & $0,739(0,652-0,837)$ \\
\hline Tercil 3 & $1,101(0,689-1,760)$ & & $0,838(0,734-0,957)$ & $1,992(1,540-2,577)$ \\
\hline
\end{tabular}

IC95\%: intervalo de confianza del 95\%; ISV: índice sintético de vulnerabilidad; RR: riesgo relativo; VGS: variación del gasto sanitario.

Todas las agrupaciones parecieron experimentar una tendencia creciente desde 2007, exceptuando el tercer tercil.

La tabla 4 muestra los RR de muerte del periodo 2008-2013 frente al 2002-2007 según terciles de VGS y ISV, ajustados por edad. Si se tiene en cuenta únicamente la VGS, el RR de muerte solo aumentó de manera significativa del primer al segundo periodo en la agrupación correspondiente al primer tercil, un $45,7 \%$ $(\mathrm{RR}=1,457)$. Al considerar únicamente el ISV se observó un incremento del RR en el primer tercil, del 24,1\% (RR=1,241).

Al considerar ambas clasificaciones (ISV y VGS) se produjeron incrementos significativos del riesgo entre el primer y el segundo periodo en las combinaciones con tercil 1 (mayor incremento de gasto) de VGS (excepto la del tercil 3 de ISV, en la cual, aunque el RR fue superior a 1, no fue significativo), y la combinación de tercil 3 (más vulnerable) de ISV y también tercil 3 (menor incremento de gasto) de VGS, con un 99,2\% de exceso de riesgo de muerte del primer al segundo periodo.

\section{Discusión}

En este estudio se ha hallado un aumento de las muertes por causas relacionadas con la atención médica y cirugía entre el periodo anterior a la crisis económica y el periodo tras el inicio de esta, con diferencias en la magnitud y la tendencia por comunidades autónomas (tabla 1). Aunque las diferencias en las tasas no fueron significativas, para el conjunto de España se evidenció un cambio de tendencia al alza desde 2008, que fue mayor en las comunidades autónomas más vulnerables. Aquellas con menor incremento del GS mantuvieron en todos los periodos TEM superiores al resto de agrupaciones. El gasto sanitario se incrementó de forma desigual en todas las comunidades autónomas, con mayor RR de muerte en el periodo tras el inicio de la crisis que en el periodo anterior en las de mayor incremento del gasto. Al considerar conjuntamente la vulnerabilidad y el gasto sanitario, el RR fue mayor en las comunidades autónomas de mayor vulnerabilidad y menor incremento del gasto, aunque otras situaciones también produjeron elevados RR.

El indicador de vulnerabilidad mostró una distribución desigual entre las comunidades autónomas, con mayor impacto de la crisis en el litoral mediterráneo y, en general, en la zona oriental del país, con la excepción de Castilla-León y La Rioja. Este mayor impacto se debió al elevado endeudamiento y la especialización en actividades económicas de baja productividad y altamente cíclicas ${ }^{13}$.
Al estudiar la diferencia entre los periodos previo y posterior al inicio de la crisis, en conjunto se observó un incremento en el gasto per cápita, con diferencias entre comunidades autónomas. A diferencia de lo ocurrido en otros países de la Organización para la Cooperación y el Desarrollo Económico (OCDE $)^{15}$, no son las comunidades autónomas más afectadas por la crisis las que más han reducido su gasto sanitario per cápita. Informes previos sobre la situación de salud de países europeos ya mencionaban el caso de España, donde contrariamente a otros países el producto interior bruto no se correlacionó con el gasto sanitario ${ }^{16}$.

Estudios realizados sobre el efecto de la crisis en la salud muestran resultados diferentes según la población a estudio, el resultado de salud analizado, el contexto (duración y severidad de la crisis, y políticas desarrolladas) y la metodología utilizada ${ }^{15,17-19}$. En este estudio se ha evidenciado un incremento de las TEM en las comunidades autónomas con mayor vulnerabilidad, estabilizándose o disminuyendo en el resto de las comunidades autónomas. Sin embargo, al comparar ambos periodos y analizar el RR, los resultados no fueron significativos y mostraron un aumento del riesgo tanto en las comunidades que se encuentran con mayor vulnerabilidad como en las que se encuentran con menor vulnerabilidad. Diversos estudios relacionan la mortalidad con el estatus socioeconómico y muestran que los riesgos de mortalidad son más bajos en los grupos con niveles educativos más altos y que la mortalidad evitable es más alta en las clases sociales más bajas 3,20,21. Por otro lado, un estudio en el que se analizó la seguridad del paciente en diversos países de la Unión Europea asoció un menor número de efectos adversos con un bajo nivel socioeconómico, explicado por un menor acceso de este colectivo a los servicios de salud y una menor exposición a sufrir estos daños ${ }^{22}$.

En cuanto a la TEM según el gasto sanitario per cápita, únicamente las comunidades autónomas con mayor incremento del gasto presentan un incremento del riesgo de mortalidad tras el inicio de la crisis económica, aunque su TEM sigue muy por debajo de las comunidades autónomas con menor incremento del gasto. En consecuencia, los resultados obtenidos sugieren que un menor gasto sanitario per cápita podría asociarse con mayores TEM, aunque los cambios tras el inicio de la crisis económica se relacionarían con cambios en las TEM solo en el grupo de mayor incremento del gasto. En general, los estudios que relacionan el gasto sanitario con diferentes resultados de salud muestran una asociación significativa entre ellos ${ }^{23-26}$, aunque también sugieren que esta asociación 
disminuye con niveles altos de gasto sanitario ${ }^{27}$ y varía según las partidas presupuestarias estudiadas ${ }^{14}$. Los resultados obtenidos en el presente estudio podrían explicarse por estos factores, y principalmente por la utilización del gasto sanitario per cápita total con distribución desigual por comunidades autónomas. Un estudio sobre la asociación entre el gasto sanitario y la seguridad del paciente concluyó que existen numerosos factores de confusión en el análisis, identificando la organización del gasto sanitario como un factor importante que debe tenerse en cuenta ${ }^{22}$.

Este estudio sugiere que las variables socioeconómicas podrían ser un importante factor de confusión en el análisis de la asociación entre el gasto sanitario y los resultados de salud. Cuando se agruparon las comunidades autónomas teniendo en cuenta la vulnerabilidad y el incremento del gasto, los resultados fueron diferentes a los obtenidos por separado. Aquellas comunidades con mayor vulnerabilidad y menor incremento del gasto per cápita tuvieron un mayor incremento del riesgo de mortalidad tras el inicio de la crisis. Este resultado es similar al encontrado en un estudio realizado por el Grupo de Trabajo de Salud de la OCDE en 2013, en el que se analizó el vínculo entre desempleo y diferentes indicadores de salud. En aquellos países donde la reducción del gasto había sido más importante, el desempleo se asociaba a peores indicadores de utilización de los servicios y de seguridad del paciente ${ }^{15}$.

Este estudio tiene las limitaciones propias de un estudio ecológico, por lo que resulta difícil establecer una relación causal y no refleja la realidad individual, principalmente en zonas de grandes desigualdades. En cuanto a los riesgos relativos estimados entre periodos, debido al bajo número de defunciones por la causa estudiada han sido estimados agregando comunidades autónomas en forma de terciles (de gasto, de vulnerabilidad o de ambos), y no por comunidades autónomas, si bien podría haberse utilizado otro tipo de modelizaciones.

Por otro lado, diversos estudios muestran una discrepancia en el registro de las causas subyacentes de muerte, siendo de las más afectadas las analizadas en este estudio ${ }^{28,29}$. Sin embargo, estas discrepancias no afectarían al análisis en el tiempo y en las diferentes comunidades autónomas. El gasto sanitario per cápita global nos da una visión muy general y por sí solo no puede explicar las diferencias de calidad en la asistencia sanitaria en las comunidades autónomas. En referencia a la construcción de los indicadores de impacto de la crisis, responden a criterios no arbitrarios y de cierta solidez, y permiten ofrecer una visión del impacto territorial de la crisis, sin descartar posibles matizaciones o mejoras. La agrupación de las comunidades autónomas por terciles de incremento de gasto o vulnerabilidad ha respondido a un criterio eminentemente estadístico. Podrían haberse utilizado otros criterios, por ejemplo puntos de corte fijos preestablecidos.

\section{Conclusiones}

El número de muertes por complicaciones médicas y quirúrgicas aumentó para el conjunto de España en el periodo estudiado, si bien las TEM no se modificaron sustancialmente, aunque con diferencias por comunidades autónomas. Se produjeron diferencias en el incremento del gasto sanitario y en el índice de vulnerabilidad por comunidades autónomas entre el periodo anterior y posterior al inicio de la crisis económica.

A pesar de que la relación entre el incremento en la inversión pública en salud y la disminución de la mortalidad por complicaciones debidas a la atención médica o cirugía no ha quedado claramente demostrada, sí ha podido determinarse que aquellas comunidades autónomas con menor incremento del gasto sanitario antes y después del inicio de la crisis económica presentaron TEM superiores al resto a lo largo de todo el periodo de estudio, y que las comunidades autónomas más vulnerables a la crisis y con menor incremento del gasto presentaron el mayor incremento del riesgo de muerte entre periodos. Dado el aumento en las muertes y su vínculo con fallos evitables del sistema sanitario que podrían reducirse tomando las medidas adecuadas, es necesario seguir investigando sobre estas causas de mortalidad.

\section{¿Qué se sabe sobre el tema?}

En los países de elevados ingresos, la mortalidad evitable tratable ha venido descendiendo en los últimos años. En España, poco se sabe sobre la evolución de la mortalidad tratable por "complicaciones debidas a la atención médica o cirugía». La crisis económica de 2008 podría haber modificado las condiciones de trabajo y asistenciales que pueden influir en el riesgo de muerte por estas causas.

\section{¿Qué aporta el estudio realizado a la literatura?}

Tras el inicio de la crisis económica de 2008 se ralentizó el gasto sanitario y aumentaron las muertes por estas causas, con un mayor incremento del riesgo de muerte en las comunidades autónomas con mayor impacto de la crisis y menor incremento del gasto sanitario. Se hace evidente la importancia de vigilar la mortalidad tratable y orientar las políticas económicas y de salud pública a fin de evitar estas muertes evitables debidas a fallos del sistema.

\section{Editor responsable del artículo}

María-Victoria Zunzunegui.

\section{Declaración de transparencia}

El autor principal (garante responsable del manuscrito) afirma que este manuscrito es un reporte honesto, preciso y transparente del estudio que se remite a GACETA SANITARIA, que no se han omitido aspectos importantes del estudio, y que las discrepancias del estudio según lo previsto (y, si son relevantes, registradas) se han explicado.

\section{Contribuciones de autoría}

A. Nolasco y J. Moncho participaron en la concepción y el diseño del estudio. E. Vicent, A. Nolasco y J. Moncho prepararon las bases de datos para el análisis y redactaron la primera versión del manuscrito. P. Caballero-Pérez y P. Pereyra-Zamora participaron en el desarrollo del estudio y el análisis de los datos, así como en la revisión crítica del borrador con contribuciones relevantes para la versión final.

\section{Agradecimientos}

Los autores manifiestan su agradecimiento a Ricardo Méndez Gutiérrez del Valle, Luis D. Abad y Carlos Echaves, por su conformidad y autorización a hacer uso de los indicadores de vulnerabilidad de su excelente trabajo.

\section{Financiación}

Proyecto «Crisis económica y mortalidad general y tratable en España», Vicerrectorat d'Investigació i Transferència de Coneixement, Universitat d'Alacant (GER16-14). 


\section{Conflictos de intereses}

\section{Ninguno.}

\section{Anexo. Material adicional}

Se puede consultar material adicional a este artículo en su versión electrónica disponible en doi:10.1016/j.gaceta.2018.07.017.

\section{Bibliografía}

1. Nolte E, McKee CM. Measuring the health of nations: updating an earlier analysis. Health Aff. 2008;27:58-71.

2. Nolte E, McKee M. Variations in amenable mortality - trends in 16 high-income nations. Health Policy (New York). 2011;103:47-52.

3. Gianino MM, Lenzi J, Muça A, et al. Declining amenable mortality: time trend (2000-2013) and geographic area analysis. Health Serv Res. 2017:52:1908-27.

4. Manderbacka K, Arffman M, Sund R, et al. Multiple social disadvantage does it have an effect on amenable mortality: a brief report. Int J Equity Health. $2014 ; 13: 1-7$

5. Gispert Magarolas R, Barés Marcano MA, Freitas Ramírez A, et al. Health system interventions assessment in Spain: an approach through the analysis of the time trends and the geographical variability of avoidable mortality between 19862001. Rev Esp Salud Publica. 2006;80:139-55

6. Plug I, Hoffmann R, Artnik B, et al. Socioeconomic inequalities in mortality from conditions amenable to medical interventions: do they reflect inequalities in access or quality of health care? BMC Public Health. 2012;12:1.

7. Mackenbach JP, Hoffmann R, Khoshaba B, et al. Using "amenable mortality" as indicator of healthcare effectiveness in international comparisons: results of a validation study. J Epidemiol Community Health. 2013;67:139-46.

8. Ministerio de Sanidad, Servicios Sociales e Igualdad. Manual de codificación CIE10-ES Diagnósticos. Vol. 1. Clasificación Internacional de Enfermedades. 2016 p. 303. (Consultado el 1/9/2017.) Disponible en: http://www.msssi.gob.es/estad Estudios/estadisticas/normalizacion/CIE10/UT_MANUAL_DIAG_2016_prov1.pdf

9. Ministerio de Sanidad, Servicios Sociales e Igualdad. Estrategia de seguridad del paciente del Sistema Nacional de Salud 2015-2020. Disponible en: https://www.seguridaddelpaciente.es/resources/documentos/2015/Estrategia Seguridad del Paciente 2015-2020.pdf

10. Aranaz JM, Aibar Remón C, Vitaller Burillo J, et al. Estudio Nacional sobre los Efectos Adversos ligados a la Hospitalización. ENEAS 2005. Madrid: Ministerio de Sanidad y Consumo; 2006.

11. Aranaz JM, Aibar C, Galán A et al. La asistencia sanitaria como factor de riesgo: los efectos adversos ligados a la práctica clínica. Gac Sanit. 2006;20( Supl 1):41-7.

12. López-Casasnovas G. La crisis económica española y sus consecuencias sobre el gasto social. Informe SESPAS 2014. Gac Sanit. 2014·28( Supl 1):18-23.
13. Méndez R, Abad LD, Echaves C. Atlas de la crisis: impactos socioeconómicos y territorios vulnerables en España. Valencia: Tirant lo Blanch; 2015.

14. Ministerio de Sanidad, Servicios Sociales e Igualdad. Estadística de gasto sanitario público. Principio de Devengo (2002-2015). (Consultado el 6/3/2016.) Disponible en: http://www.msssi.gob.es/estadEstudios/estadisticas/ inforRecopilaciones/gastoSanitario2005/home.htm

15. Van Gool K, Pearson M. Health, austerity and economic crisis: assessing the short-term impact in OECD countries. OECD Health Working Papers; . 2014

16. OECD. Health at a glance: Europe 2010. ECD; 2012. Disponible en: http://www oecd-ilibrary.org/social-issues-migration-health/health-at-a-glance-europe2012_9789264183896-en

17. Karanikolos M, Heino P, McKee M, et al. Effects of the global financial crisis on health in high-income OECD countries. Int J Heal Serv. 2016;46:208-40.

18. Tapia Granados JA. La crisis y la salud en España y en Europa: ¿está aumentando la mortalidad? Salud Colect. 2014:10:81-91.

19. Cortès I, Gonzàlez B. Crisis económica financiera y salud en España. Evidencia y perspectivas. Informe SESPAS 2014. Gac Sanit. 2014;28( Supl 1):1-6.

20. Castelli A, Nizalova O. Avoidable mortality: what it means and how it is measured. CHE; 2011 (CHE Discussion Papers). Report No.: Research Paper 63. Disponible en: https://www.york.ac.uk/media/che/documents/papers/researchpapers/ CHERP63_avoidable_mortality_what it means and how_it_is_measured.pdf

21. Nolasco A, Quesada JA, Moncho J, et al. Trends in socioeconomic inequalities in amenable mortality in urban areas of Spanish cities, 1996-2007. BMC Public Health. 2014;14:1-12.

22. Filippidis FT, Mian SS, Millett C. Perceptions of quality and safety and experience of adverse events in 27 European Union healthcare systems, 2009-2013. Int J Qual Heal Care. 2016;28:721-7.

23. Budhdeo S, Watkins J, Atun $\mathrm{R}$, et al. Changes in government spending on healthcare and population mortality in the European Union, 1995-2010: a cross-sectional ecological study. J R Soc Med. 2015;108: 490-8.

24. Heijink R, Koolman X, Westert GP. Spending more money, saving more lives? The relationship between avoidable mortality and healthcare spending in 14 countries. Eur J Heal Econ. 2013;14:527-38.

25. Hiam L, Dorling D, Harrison D, et al. What caused the spike in mortality in England and Wales in January 2015? J R Soc Med. 2017;110:131-7.

26. Maruthappu M, Ng KYB, Williams C, et al. The association between government healthcare spending and maternal mortality in the European Union, 1981-2010: a retrospective study. BJOG. 2015:122:1216-24.

27. Organization for Economic Cooperation and Development. Health at a glance 2015, OECD Indicators. OECD Publ; 2015. (Consultado el 30/1/2018.) Disponible en: http://apps.who.int/medicinedocs/documents/s22177en/s22177en.pdf

28. Johansson LA, Westerling R. Comparing hospital discharge records with death certificates: can the differences be explained? J Epidemiol Community Health. 2002;56:301-8.

29. Pederson A, Ellingson C. Data quality in the causes of death registry Tidsskrift for Den norske legeforening. Tidsskr Nor Legeforen. 2015:768-70. Disponible en: http://tidsskriftet.no/article/3325643/en_GB 